\title{
Outage Minimization and Optimal Power Control for the Fading Relay Channel
}

\author{
Nasir Ahmed \\ Dept. of Electrical and Computer \\ Engineering \\ Rice University \\ Houston, TX, 77005 \\ e-mail: nasir@rice.edu
}

\author{
Mohammad Khojastepour \\ Dept. of Electrical and Computer \\ Engineering \\ Rice University \\ Houston, TX, 77005 \\ e-mail: amir@rice.edu
}

\author{
Behnaam Aazhang \\ Dept. of Electrical and Computer \\ Engineering \\ Rice University \\ Houston, TX, 77005 \\ e-mail: aaz@rice.edu
}

\begin{abstract}
In this work, we show that in the wireless relay network, a tremendous savings in energy can be achieved by having side information at the transmitters and by employing power control. We present efficient protocols and the corresponding optimal power control policies that approach the universal lower bound on the outage probability of the block fading relay channel. Each of the proposed protocols have their own utility for specific channel conditions. However, a hybrid protocol between two known coding schemes is the best scheme for all channel conditions and is sufficient to approach the lower bound on outage probability. Unlike the single link channel, we show that exploiting the knowledge of the channel at the transmitters can significantly lower the outage even if the transmit powers at the source and relay have to be kept constant. In this case, it is also demonstrated that the lower bound on outage is closely followed by the outage probability of the hybrid protocol. Our results reveal that exploiting the right network protocol in conjunction with power control result in orders of magnitude savings in power over direct transmission for a target performance level.
\end{abstract}

\section{INTRODUCTION}

Since its proposition more than 30 years ago in [7], the capacity of the relay channel has been an open problem even for the case of Gaussian channels. In the fading environment, where the use of relaying has been shown to increase throughput, there is even less knowledge regarding efficient network coding protocols. In this work, we consider the block fading channel, and with outage probability as the performance metric, we investigate methods to improve the frame error rate performance by analyzing efficient protocols and through optimal power control. To capture the frame error rate improvements, we consider the outage probability as the performance metric, since it serves as a lower bound to the frame error rate in the fading channel for large block lengths.

The main contribution of this work is to address the outage minimization problem with channel state information at the transmitters. First, if the source and relay must transmit with a constant power, then with the use of channel state information, they can allocate for phase offsets to ensure that the signals at the receiver add coherently. Additionally, for different channel states, the source and relay can modify the correlation between their transmitted signals to further reduce the outage. Under these assumptions, a hybrid between two known network codes is shown to be sufficient to approach the lower bound on outage probability.

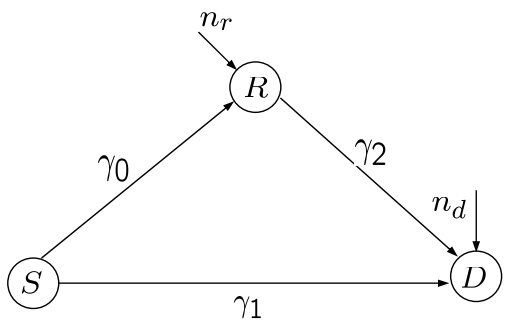

Fig. 1: Layout of the relay network.

Second, when channel knowledge is available and the source and relay can modify their power from one time slot to the next, we derive the optimal power control policy. The power control procedure is applied to many known network codes and the tremendous gains over constant power transmission are shown. A hybrid between two known coding schemes is once again shown to provide performance close to the lower bound on outage probability for the Rayleigh fading channel with power control.

The results in this paper motivate the need for efficient protocols to allow for feedback regarding channel state information in the network setting. With power control, the savings in energy through optimal power allocation can lead to increased battery life and throughput for mobiles. In fact, even with limited feedback, the gains of using power control in the network can lead to tremendous savings in power [1].

The rest of the paper is organized as follows. Section II provides background regarding the protocols and models used throughout the paper. Section III describes the transmission protocols for the relay channel that are studied in this work. Section IV investigates the outage performance of the relay protocols under the assumption of channel knowledge being available to the transmitters. The first case considered is constant power transmission and in Section IV.B, the optimal power allocation is shown. Section V concludes the paper.

\section{Preliminaries}

A. Network Model: In this work, communication occurs over a relay network, with one relay node and one sourcedestination pair, as is shown in Figure 1. The relay $R$ assists in the communication of data between the source $S$ and the destination $D$. It is assumed that link $i$ in the network is attenuated by fading coefficient $h_{i}$, where $i \in\{0,1,2\}$. The magnitudes of these coefficients are assumed to follow a Rayleigh distribution. At both the source and relay, the received signal is corrupted by additive white Gaussian noise with unit variance.

In the sequel, we will denote $\gamma_{0}=\left|h_{0}\right|^{2}, \gamma_{1}=\left|h_{1}\right|^{2}$ and $\gamma_{2}=\left|h_{2}\right|^{2}$, as can be seen in Figure 1. The network channel 


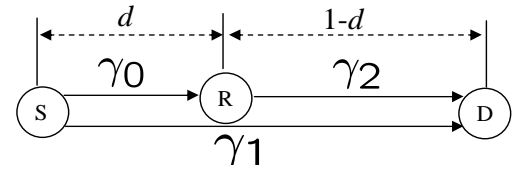

Fig. 2: Layout of the relay network with the relay node located along a straight line from the source to the destination.

state is defined by the 3-tuple $\underline{\gamma}=\left(\gamma_{0}, \gamma_{1}, \gamma_{2}\right)$, where $\gamma_{i}$ follows an exponential distribution with mean $\lambda_{i}, i \in\{0,1,2\}$. The parameter $\lambda_{i}$ captures the pathloss across link $i$ in the network, which is a function of the length of the link, and the pathloss exponent $\alpha$. In practical applications, typically, $\alpha$ lies in the range $(2,5)$. As can be seen in Figure 1, the destination has the ability to transmit feedback information to both the source and relay, and this channel knowledge can be used to perform power control. It is assumed that the feedback links are noiseless.

B. Performance Analysis: We consider a block fading model, where the fading coefficients $\gamma_{i}$ are constant over a block and are independent from one block to the next. A practical analysis tool for the block fading environment is the outage probability. Outage probability is the probability that the instantaneous achievable rate of the channel is less than the transmission rate, or in other words,

$$
P_{\text {out }}\left(R, P_{s}, P_{r}\right)=\operatorname{Prob}\left[R>R_{g e n}\left(\underline{\gamma}, P_{s}, P_{r}\right)\right],
$$

where $R_{\text {gen }}$ is the achievable rate of the transmission protocol. In (1), $P_{s}$ is the transmit power of the source, $P_{r}$ is the transmit power of the relay and $R$ is the attempted rate of transmission.

To consider different relay situations, we consider the model shown in Figure 2. It is assumed that the distance between the source and relay is one, and the relay is located in a line between the source and destination. The parameter $d$ represents the distance from the source to the relay, and $1-d$ is the distance from the relay to the destination. The mean value of the fading distribution for the source-relay link is consequently $\lambda_{0}=\frac{1}{d^{\alpha}}$ and for the relay-destination link we have $\lambda_{2}=\frac{1}{(1-d)^{\alpha}}$. For the remainder of this work, we consider the model of Figure 2 to capture the effect of relay distance on performance.

\section{Relaying Protocols}

In this section, we describe the efficient relaying protocols studied in this work. In general, many network coding options are available, based on the physical limitation of the relay node and the complexity of coding allowed. An example of such a limitation is the problem of 'cheap' relay nodes, introduced in [2], where transmission and reception simultaneously in the same frequency band is not possible. In this case, a practical transmission protocol is the amplify and forward (AF) technique, developed in [4]. Given a source with average power $P_{s}$ and a relay with average power $P_{r}$, the achievable rate of the AF transmission protocol is [4]

$$
\begin{aligned}
& R_{A F}\left(\underline{\gamma}, P_{s}, P_{r}\right)= \\
& \frac{1}{2} \log \left(1+2 \gamma_{1} P_{s}+\frac{4 \gamma_{2} P_{s} \gamma_{0} P_{r}}{1+2 P_{s} \gamma_{0}+2 P_{r} \gamma_{2}}\right) .
\end{aligned}
$$

Note that in (2), since each transmitter sends data for half the time slot, the source uses power $2 P_{s}$ and the relay uses power $2 P_{r}$ to guarantee an average power of $P_{s}+P_{r}$ per time slot.
For relay nodes that can transmit and receive simultaneously, protocols with higher achievable rates are available. The limits of communication on the relay channel are defined by the cut-set upper bound(UB)[5]. When knowledge of the network channel state $\gamma$ is available at the transmitters, the upper bound on the achievable rate is

$$
\begin{aligned}
& R_{U B}\left(\underline{\gamma}, P_{s}, P_{r}\right)= \\
& \max _{0 \leq \rho \leq 1} \min \left\{\log \left(1+\left(1-\rho^{2}\right)\left(\gamma_{0}+\gamma_{1}\right) P_{s}\right),\right. \\
& \left.\log \left(1+\gamma_{1} P_{s}+\gamma_{2} P_{r}+2 \rho \sqrt{\gamma_{1} \gamma_{2} P_{s} P_{r}}\right)\right\} .
\end{aligned}
$$

The parameter $\rho$ controls the correlation between the signals transmitted by the source and relay, and changes with different channel states. In (3), it is assumed that the transmitters offset their signals to correct for the phase to ensure that the signals at the receiver add coherently. The rate of (3) is an upper bound, and no coding schemes have been found that have this rate. One final point regarding (3) is that in terms of outage probability, this would lead to a lower bound.

In terms of achievable schemes, under the assumption that the relay fully decodes the transmission from the source, an achievable rate was derived in [5] and shown to be

$$
\begin{array}{r}
R_{D F}\left(\underline{\gamma}, P_{s}, P_{r}\right)=\max _{0 \leq \rho \leq 1} \min \left\{\log \left(1+\left(1-\rho^{2}\right) \gamma_{0} P_{s}\right)\right. \\
\left.\log \left(1+\gamma_{1} P_{s}+\gamma_{2} P_{r}+2 \rho \sqrt{\gamma_{1} \gamma_{2} P_{s} P_{r}}\right)\right\}
\end{array}
$$

Once gain, in (4), the parameter $\rho$ is chosen based on the current channel state, and that the source and relay use the knowledge of the phase offset of the channel to ensure coherent reception at the destination. One limitation of (4) is that if $\gamma_{1}>\gamma_{0}$, then direct transmission offers a higher rate. However, instead of decoding, the relay can forward an estimate of its received signal $[3,5]$. This protocol, called estimate and forward [3], always achieves a higher rate than direct transmission. The achievable rate of this scheme is

$$
\begin{aligned}
& R_{E F}\left(\underline{\gamma}, P_{s}, P_{r}\right)= \\
& \log \left(1+P_{s} \gamma_{1}+\frac{P_{s} \gamma_{0} P_{r} \gamma_{2}}{1+P_{s} \gamma_{0}+P_{s} \gamma_{1}+P_{r} \gamma_{2}}\right) .
\end{aligned}
$$

When the relay is positioned near the source, then the DF protocol performs well, while the EF protocol has a high achievable rate for relays that are close to the destination. With this in mind, we propose an adaptive protocol which chooses the protocol with the larger achievable rate in each time slot. The proposed transmission scheme, which is a hybrid between the estimate and forward and the decode and forward transmission schemes, has an achievable rate as follows

$$
\begin{aligned}
& R_{H B}\left(\underline{\gamma}, P_{s}, P_{r}\right)= \\
& \max \left\{R_{E F}\left(\underline{\gamma}, P_{s}, P_{r}\right), R_{D F}\left(\underline{\gamma}, P_{s}, P_{r}\right)\right\} .
\end{aligned}
$$

As will be seen, this hybrid protocol has an interesting property that it is able to perform well in cases where both the EF and DF protocols perform poorly.

\section{Outage Minimization with Channel State INFORMATION AT THE TRANSMITTER AND RECEIVER}

A. Constant Power Transmission: In a relay system, even when the source and relay are restricted to transmit with a constant power in each time slot, methods exist to reduce 


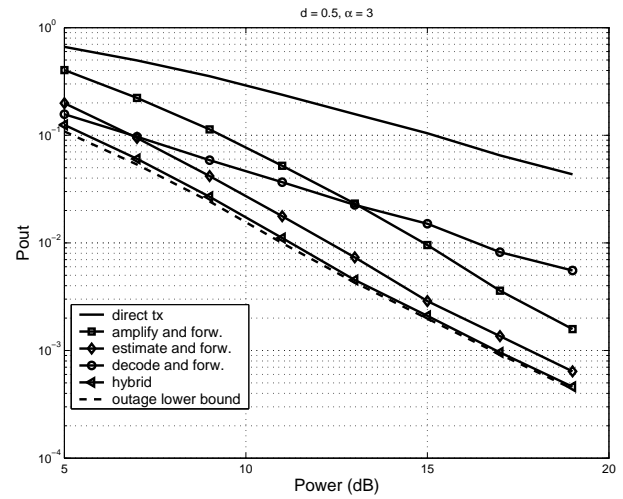

Fig. 3: Probability of outage vs. sum network power for various relaying protocols using constant power and a rate $\mathrm{R}=1, d=0.5$, and $\alpha=3$. It is assumed that the source and relay have equal power constraints.

the outage probability, which is not the case for a direct transmission system. When channel state information is available to the source and relay, two optimizations can be performed. First, the phase at the source and relay can be corrected, such that the signals at the destination node combine coherently. Second, for some network coding protocols, the correlation between the signals from the source and relay can be adjusted to maximize the rate in each channel state. Both of these optimizations can be performed while performing constant power transmission.

Figure 3 shows the outage probability results for the case of $d=0.5$, where $d$ is the distance parameter from Figure 2, with the source and relay able to use phase correction and select the optimal $\rho$ for each transmission. It is assumed that the source and relay both have the same average power constraint, and the plots are versus $2 P_{a v g}$ for comparison. The results for the direct link system using a source power of $2 P_{a v g}$ is shown as a baseline for comparison. Since the decode and forward protocol suffers from decoding errors at the relay, it also has the same diversity order as direct link transmission, although a better coding gain. The amplify and forward protocol has a second order diversity, yet it has poor performance at low powers. This protocol suffers from the fact that the source and relay remain idle for half of each transmission slot. The estimate and forward is also seen in the figure, and it has a $2.5 \mathrm{~dB}$ advantage over amplify and forward. Amazingly, the hybrid protocol is shown in the figure to closely follow the outage lower bound (from (3)) for a relay channel with constant power. This confirms that from an outage perspective with constant power transmission, the hybrid protocol is sufficient to approach the fundamental limits.

Using a distance of $d=0.5$ indicates that the relay is midway between the source and relay. However, it is interesting how robust the transmission schemes are to the relay node's position. In Figure 4, for a fixed sum network power of $10 \mathrm{~dB}$, the outage probability is shown as a function of $d$. The amplify and forward protocol performs well when the relay is located midway between the source and destination, but performance degrades when $d$ is small or large. For a good source-relay link, which occurs when $d$ is small, the decode and forward protocol performs well, as the chance of outage on this link is low. As the relay moves towards the destination, the performance degrades substantially. The estimate and forward pro-

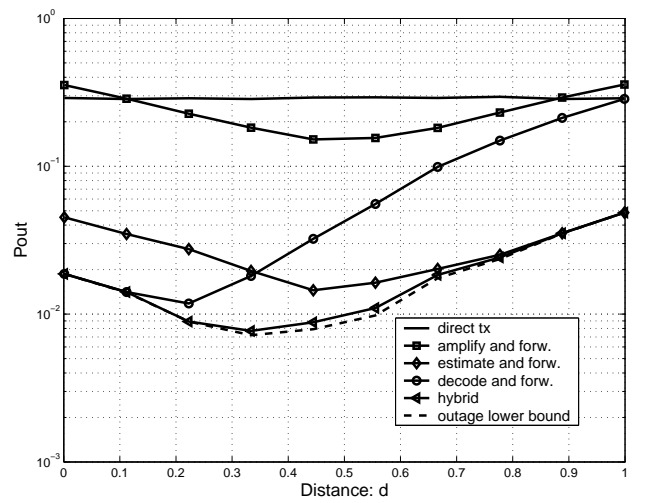

Fig. 4: Probability of outage vs. relay distance to source for various relaying protocols using constant power and a rate $\mathrm{R}=1$, and $\alpha=3$ with a sum network power of $10 \mathrm{~dB}$. Source and Relay have equal power constraints.

tocol exhibits almost the opposite behavior. For relays that are closer to the source, it performs poorly, while as the relaydestination link reduces, the performance approaches the optimal solution. Finally, the hybrid protocol is also shown in Figure 4, and it can be seen that throughout nearly the entire range of $d$, the outage performance follows closely to the lower bound on outage probability. This indicates that the hybrid protocol is robust to node positioning. The power of the HB protocol becomes clearer by looking at distance $\mathrm{d}=0.33$, where both the EF and DF protocols are far from the lower bound, but the HB still closely follows the bound. By using a combination of the DF and EF protocols, the HB protocol can compensate for their mutual weakness.

B. Outage Minimization with Power Control: When the network channel state is available at the source and relay, outage minimization with power control can provide significant performance improvements. Given a network channel state of $\gamma$, the source transmits with power $P_{s}(\gamma)$ and the relay with $\overline{P_{r}}(\gamma)$. To obtain significant reductions in outage probability, the minimization is done with respect to an average sum power constraint, meaning that

$$
E_{\underline{\gamma}}\left[P_{s}(\underline{\gamma})+P_{r}(\underline{\gamma})\right] \leq 2 P_{a v g}
$$

The network power optimization problem involves the minimization of outage subject to a sum power constraint with two variables $P_{s}$ and $P_{r}$, which seems intractable. However, we next show that this problem can be turned into a single variable optimization problem which allows us to use the same idea of outage minimization used for the single link fading channel [6]. This leads to the optimal power control policy for the relay channel.

In general, the procedure for minimizing the outage probability involves two steps, as was shown in [6]. The first step requires the solution to a short term power allocation, which minimizes the network power and guarantees zero outage in each network channel state while transmitting at the target spectral efficiency. However, with network power control, in the solution of the short term power, an additional optimization must be performed to maximize the achievable rate by finding the optimal values of $P_{s}$ and $P_{r}$ given a constraint on their sum. Second, a cutoff region is found that modifies the short term power allocation to shut off transmission in poor 
channel conditions. The cutoff region is determined to satisfy the average sum power constraint.

Given the instantaneous value of the network channel state, $\gamma$, the source transmits with power $P_{s}(\gamma)$ and the relay with power $P_{r}(\gamma)$. Assuming a generic transmission protocol with an achievable rate of $R_{g e n}\left(\underline{\gamma}, P_{s}(\underline{\gamma}), P_{r}(\underline{\gamma})\right)$, the outage minimization procedure becomes

$$
\begin{array}{r}
P_{\text {out }}(R, \underline{\gamma})=\operatorname{Prob}\left(R_{\text {gen }}\left(\underline{\gamma}, P_{s}(\underline{\gamma}), P_{r}(\underline{\gamma})\right)<R\right)= \\
E_{\underline{\gamma}}\left[\mathcal{I}_{F}\left\{R_{\text {gen }}\left(\underline{\gamma}, P_{s}(\underline{\gamma}), P_{r}(\underline{\gamma})\right)<R\right\}\right],
\end{array}
$$

where $\mathcal{I}_{F}(\cdot)$ is the indicator function. The objective of the power control algorithm is to minimize the outage probability subject to the constraint that $E_{\gamma}\left[P_{s}(\gamma)+P_{r}(\gamma)\right] \leq 2 P_{\text {avg }}$. It can be rigorously shown that the minimization of (8) with a long-term sum power constraint requires the solution of a short term power constraint $P_{s}(\gamma)+P_{r}(\gamma)=2 P_{s t}^{*}(\gamma)$ for some short-term power $P_{s t}^{*}(\underline{\gamma})$ depending on the network state $\underline{\gamma}$.

The optimal power allocation with a long term constraint has the following structure [6]

$$
P^{*}(\underline{\gamma})=\left\{\begin{array}{l}
P_{s t}^{*}(\underline{\gamma}), \text { with probability } w(\underline{\gamma}) \\
0, \text { with probability } 1-w(\underline{\gamma}) .
\end{array}\right.
$$

The minimized outage probability is then $P_{\text {out }}(R, \underline{\gamma})=E_{\gamma}[1-$ $w(\gamma)$ ]. To obtain $P_{s t}^{*}(\gamma)$, we find a power control policy with minimum power that guarantees zero outage at transmission rate $R$. Note that for each $\underline{\gamma}$, a $P_{s}(\underline{\gamma})$ and a $P_{r}(\underline{\gamma})$ exist which maximize the achievable rate for a particular sum power $P_{s t}(\gamma)$. If we let

$$
\begin{array}{r}
G\left(\underline{\gamma}, P_{s t}(\underline{\gamma})\right)=\max _{P_{s}(\underline{\gamma}), P_{r}(\underline{\gamma})}\left\{R_{g e n}\left(\underline{\gamma}, P_{s}(\underline{\gamma}), P_{r}(\underline{\gamma})\right):\right. \\
\left.P_{s}(\underline{\gamma})+P_{r}(\underline{\gamma}) \leq 2 P_{s t}(\underline{\gamma})\right\},
\end{array}
$$

then for a fixed $P_{s t}(\underline{\gamma}),(10)$ can be written as

$$
\begin{gathered}
G\left(\underline{\gamma}, P_{s}(\underline{\gamma})\right)=\max _{P_{s}(\underline{\gamma})}\left\{R_{g e n}\left(\underline{\gamma}, P_{s}(\underline{\gamma}), 2 P_{s t}(\underline{\gamma})-P_{s}(\underline{\gamma})\right)\right. \\
\left.: 0 \leq P_{s}(\underline{\gamma}) \leq 2 P_{s t}(\underline{\gamma})\right\} .
\end{gathered}
$$

The maximization can be done over the single variable $P_{s}(\gamma)$. Note that with the use of (11), the original optimization over two variables $P_{s}(\underline{\gamma})$ and $P_{r}(\underline{\gamma})$ is now turned into a single variable maximization over $P_{s}(\bar{\gamma})$ given the sum power constraint $2 P_{s t}(\gamma)$. Now, for any $P_{s t}(\bar{\gamma})$, the optimal allocation between the source and relay is known that maximizes the achievable rate. The next step is to determine the optimal value of $P_{s t}^{*}(\gamma)$.

To solve the short term power function $P_{s t}^{*}(\gamma)$, the minimum $P_{s t}(\gamma)$ that satisfies the rate constraint is determined. The optimal short term power, $P_{s t}^{*}(\gamma)$, can be obtained as the solution to

$$
P_{s t}^{*}(\underline{\gamma})=\min _{P_{s t}(\underline{\gamma})} G\left(\underline{\gamma}, P_{s t}(\underline{\gamma})\right) \geq R .
$$

This corresponds to the minimum power for zero outage, given that the source and relay power are optimally calculated to maximize the achievable rate.

The policy $P_{s t}^{*}(\underline{\gamma})$ guarantees zero outage for each channel state $\gamma$. To ensure that the long term power constraint is satisfied, a weighting function $w(\underline{\gamma})$ is found as the solution to

$$
\max _{w}\left\{E\left[P_{s t}^{*}(\underline{\gamma}) w(\underline{\gamma})\right] \leq P_{a v g}, 0 \leq w(\underline{\gamma}) \leq 1\right\} .
$$

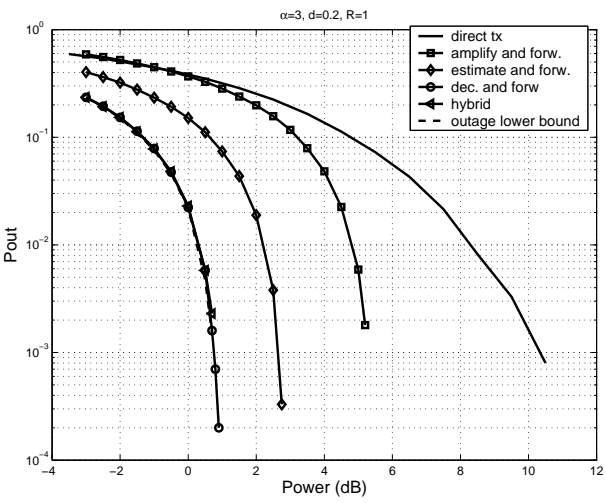

Fig. 5: Probability of outage vs. network power for $\alpha=3, \mathrm{R}=1$, and $\mathrm{d}=0.2$

Following the discussion of [6], the optimal value of $w(\gamma)$ has the form $[8]$

$$
w(\underline{\gamma})=\left\{\begin{array}{l}
1, \text { if } P_{s t}^{*}(\underline{\gamma})<s^{*} \\
w_{0}, \text { if } P_{s t}^{*}(\underline{\gamma})=s^{*} \\
0, \text { if } P_{s t}^{*}(\underline{\gamma})>s^{*}
\end{array}\right.
$$

with $w_{0} \in(0,1)$ and

$$
s^{*}=\sup \left\{s: \int_{\mathcal{R}(s)} P_{s t}^{*}(\underline{\gamma}) d F(\underline{\gamma})<P_{a v g}\right\},
$$

and

$$
\mathcal{R}(s)=\left\{\underline{\gamma}: P_{s t}^{*}(\underline{\gamma})<s\right\} .
$$

The region $\mathcal{R}(s)$ is the set of all network channel states $\gamma$ that require $P_{s t}^{*}(\gamma)<s$. The outage region can be interpreted as a volume in the 3 -D space of all $\left(\gamma_{0}, \gamma_{1}, \gamma_{2}\right)$ that requires more power than $s^{*}$ to invert the channel effects.

It can be seen that minimizing outage and satisfying the average sum power constraint involves first solving a short term power allocation problem that completely inverts the effects of the channel, and this is followed by a cutoff power that guarantees the sum average power constraint. By using (11), the best allocation of power between the source and relay is determined for any given network channel state. Again, we emphasize the point that the above solution to the longterm sum power constraint heavily depends on the fact that, for any channel state $\gamma$, the short-term sum power constraint $P_{s}(\gamma)+P_{r}(\gamma)=2 P_{s t}(\bar{\gamma})$ has to be satisfied [8].

In this section, we have outlined the optimal network power allocation for a general relaying protocol. The power allocation procedure for specific protocols is similar, except the solution of $G\left(\underline{\gamma}, P_{s t}(\underline{\gamma})\right)$ varies depending on the form of the achievable rate. In the next section, an analytical expression $G\left(\gamma, P_{s t}(\gamma)\right)$ will be found for the protocols of interest in this work. For a discussion of the computation of $G\left(\underline{\gamma}, P_{s t}(\gamma)\right)$ for the various relaying protocols discussed in this work, see [8].

C. Analysis and Discussion: In Figure 5, the outage probability is shown for the optimal power control policy between the source and relay. The relay is assumed to be at a distance of $d=0.2$ (based on Figure 2), which allows for a good source-relay link. The tremendous gains of performing optimal power allocation over the fading channel are seen. 


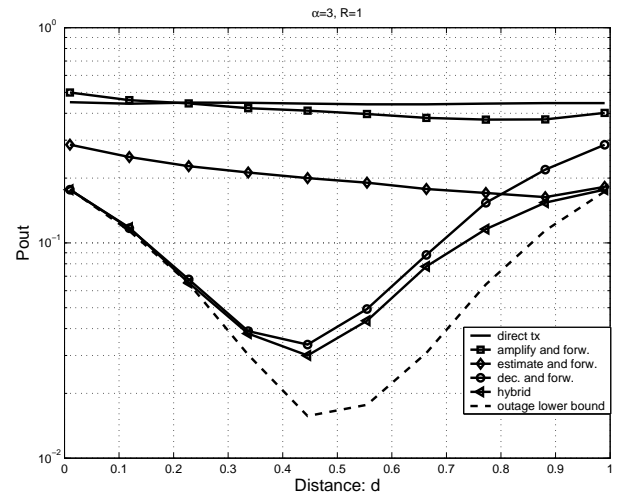

Fig. 6: Probability of outage vs. relay distance to source for various relaying protocols using constant power and a rate $\mathrm{R}=1$. The sum network power is $-1 \mathrm{~dB}$.

The lower bound on outage probability is shown, and it is apparent that the hybrid protocol and the decode and forward protocol closely follow the lower bound.

The estimate and forward protocol, on the other hand, is approximately $2 \mathrm{~dB}$ away from the lower bound. This result makes sense with what was observed for the scenario of constant power transmission, where for small values of $d$, the decode and forward and the hybrid protocols closely followed the lower bound. For large distances, however, the rate of the DF protocol degrades substantially compared to the outage lower bound. On this same figure, the outage results for the amplify and forward protocol are also plotted, and it is seen that there is a tremendous loss in performance by performing such a 'cheap' transmission protocol [2]. Finally, note that all the relaying schemes have large gains over direct transmission even with its optimal power control policy.

In Figure 6, for a fixed network power of $-1 \mathrm{~dB}$, we show the outage probability versus the distance of the relay from the source. The AF protocol has the worst performance of all the relaying schemes presented, as is expected since it falls into the category of a 'cheap' protocol. The EF technique is robust to the node position, and approaches the lower bound on outage for large d. Also, in Figure 6 it can be seen that the DF protocol is close to optimal for small $d$. The hybrid protocol can be seen to provide gains for the values of $d$ where the EF and DF protocols perform poorly, for example at $d=0.8$.

\section{Conclusions}

In this work, we have analyzed the outage performance of different relaying protocols for the fading channel with side information both at the transmitters and the receiver. The main contribution of the paper is twofold. First, having side information at the source and the relay provides a tremendous gain which can be exploited by having feedback. Moreover, even a finite rate of feedback can significantly improve the performance and lower the outage probability [1]. Second, by using a hybrid of the coding protocols discussed in [2] the universal lower bound on the outage probability can be almost achieved.

This work reveals that side information at the transmitters for the relay channel is more crucial than that of the single link channel. The side information at the transmitter can be used to devise coding schemes which perform better even if power variation is not allowed at the transmitter. However, in a single link side information at the transmitter is useless if transmitting power is constant. Additionally, in this work, we derived the optimal power allocation for different relaying protocols when power control is allowed. It is imperative to note that by exploiting power control the outage probability decays much faster. As a result, we have motivated the need for feedback information to fully realize the benefits of network coding.

\section{REFERENCES}

[1] N. Ahmed, M. Khojastepour, A. Sabharwal, and B. Aazhang Power Control with Finite Rate Feedback for Cooperative Relay Networks, Accepted for publication to IEEE ISITA 2004,

[2] M. A. Khojastepour, A. Sabharwal and B. Aazhang, "Lower Bounds on the Capacity of Gaussian Relay Channel," Submitted to the $38^{\text {th }}$ Annual Conference on Information Sciences and Systems March 17-19, Princeton, NJ., 2004.

[3] M. A. Khojastepour, A. Sabharwal and B. Aazhang, "On the Capacity of 'Cheap' Relay Networks," In Proc. 37th Annual Conf. on Info. Sciences and Systems, March 12-14, Baltimore, MD., 2003.

[4] N. Laneman, D. Tse and G. Wornell "Coooperative Diversity in Wireless Networks: Efficient Protocols and Outage Behavior," Accepted for publication to IEEE Trans. on Info. Theory April 2003.

[5] T.M. Cover and A. E. Gamal "Capacity theorems for the relay channel," IEEE Trans. on Info. Theory, vol. 25, no. 5, pp., 572-584, Sept 1979

[6] G. Caire, G. Taricco and E. Biglieri, "Optimum power control over fading channels," IEEE Trans. on Info. Theory., vol. 45, no. 5, pp., 1468-1489, July 1998.

[7] E. C. van der Meulen, "Three-terminal communication channels," Adv. Appl. Prob., vol. 3, 1971.

[8] M. A. Khojastepour, N. Ahmed, and B. Aazhang, Performance limits of the fading relay channel - Part II: Efficient protocols and the optimal power control, In Prepartion for IEEE Transactions on Information Theory 